\title{
Effect of surface condition on strain in semiconductor crystal sample
}

\author{
B.K. Serdega, E.V. Nikitenko, V.I. Prikhodenko \\ Institute of Semiconductor Physics, NAS Ukraine, 45 Prospect Nauki, Kyiv, 03028, Ukraine \\ E-mail: bshl@polarget.semicond.kiev.ua
}

\begin{abstract}
Using the optical-polarization technique with polarization modulation, we measured stresses due to surface tension in a single-crystalline silicon sample. Optical anisotropy distribution along the normal to the sample surface was studied at different state conditions, depending on its treatment. It was found that presence of a surface layer in the crystal, with physical properties differing from those in the bulk, gave rise to extended distribution of stresses in the uniform part of the crystal.
\end{abstract}

Keywords: polarization, surface, anisotropy, strain.

Paper received 09.11.00; revised manuscript received 01.02.01; accepted for publication 16.02.01.

\section{Introduction}

The optical-polarization technique (used for study and measurement of strains and stresses in semiconductor wafers, multilayer structures and integrated circuit elements) has several advantages as compared to a number of other (X-ray, optical, holographic, acoustic-resonance, etc.) methods. These advantages are, first of all, high sensitivity and spatial resolving power.

Application of this technique to measurement of such topical subject of investigation as strain resulting from surface tension, however, presents some constraints. One of them stems from the polarization transformation laws. It implies that the index ellipsoid (that characterizes crystal anisotropy due to surface tension) should be oriented in the plane that is normal to the wave vector. Clearly this condition is not fulfilled in the typical case when light is propagating along the normal to the studied surface of a thin plate. Another limitation is related to a multiple-valued dependence of the detected signal on the optical anisotropy (i.e., on stress value). This fact makes explanation of results obtained rather complicated and, sometimes, even impossible.

A different situation arises if one combines the traditional optical-polarization technique with modulation of polarization. In this case (as follows from an analysis of a role of polarization modulator in polarimetric systems [1]) the measuring system sensitivity to anisotropy (and, therefore, the dynamic range of the measured signal as well) becomes several orders greater. This fact opens up possibility for measurements of those stresses that lie in the range of the first order «interference». (This means not only the first period of the oscillating signal vs voltage curve but also the case when this dependence is strictly linear).

Here we demonstrate the above possibility by our study of stress distribution resulting from surface tension in the single-crystalline silicon samples.

\section{Experimental technique}

A cubic (side of $1 \mathrm{~cm}$ ) sample has been cut from a lowdoped $(\rho=200 \Omega \cdot \mathrm{cm}) p$-Si bar along its principal crystallographic axes. Then it was treated in a standard way to obtain mirror and flat surfaces. All the faces of the initial sample have been treated with a polishing etchant. Before further measurements the side (relative to the illuminated surface) faces were mechanically polished with a diamond paste (grain size of $2 \mu \mathrm{m}$ ) and then lapped with a powder (grain size of $40 \mu \mathrm{m}$ ).

The measured optical anisotropy was proportional to the difference $\left(\sigma_{x}-\sigma_{y}\right)$ between the principal components of stress lying in the $x-y$ plane that was perpendicular to the direction of light propagation (Fig. 1). The orientation of the crystal index ellipsoid was known since it coincided with the sample axes. So we used linear polarization of radiation in the initial state for reasons of convenience of signal registration. The azimuth orientation of polarization was determined from the transformation 


\section{B.K. Serdega et al.: Effect of surface condition on strain in semiconductor crystal sample}

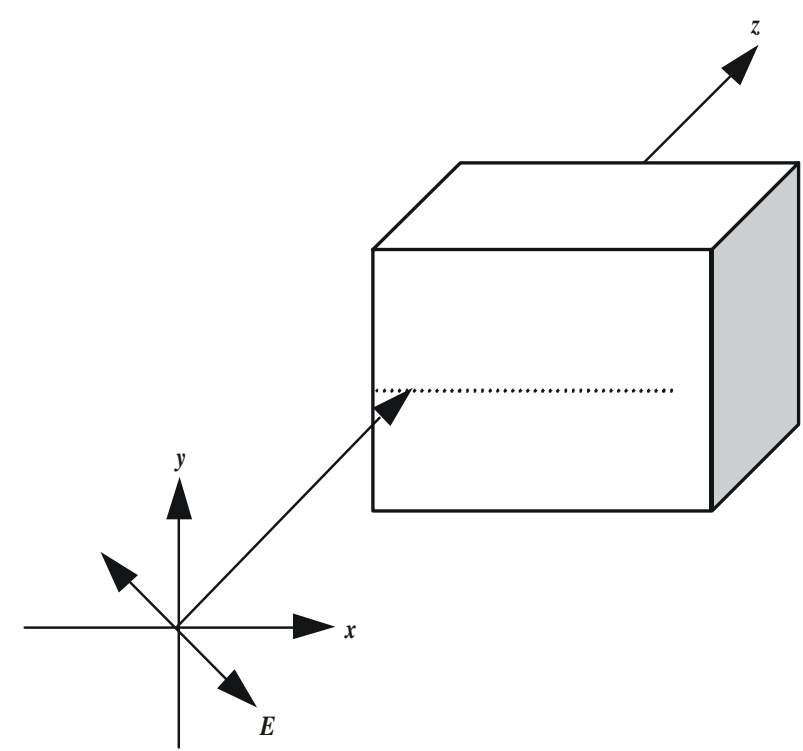

Fig. 1. Scheme of the experiment. $\boldsymbol{E}$ is the electric field of a polarized electromagnetic wave.

rules for anisotropic medium. It made an angle of $45^{\circ}$ with the sample axes and,consequently, with the optical indicatrix ones.

A He-Ne laser (wavelength $\lambda=1.15 \mu \mathrm{m}$ ) served as a source of probing radiation. Such radiation is known [2] to be weakly absorbed in Si. This fact was of importance since we used a rather thick sample. The light coming out from the sample was directed at the polarization modulator that served as a polarization analyzer. It involved the Jasperson photoelastic modulator [3] and a polarization prism whose azimuth is oriented relatively to the modulator axes in such a way as to obtain maximal alternating signal. It was registered with a lock-in nanovoltmeter tuned to measure a signal at the modulator fundamental frequency $\omega$. In this case, according to the results of the abovementioned analysis [2], one measures the circular component of (generally elliptically polarized) radiation. The dependence of this component on optical anisotropy (in other words, on stress) $\delta$ is given by the following expression: $I_{\text {out }}=I_{\text {in }} k \sin \delta \cdot \sin \omega t$. Here $I_{\text {in }}\left(I_{\text {out }}\right)$ is the intensity of light that is incident on (coming out from) the sample; $k$ is a constant that characterizes intensity attenuation by the optical system elements. One can see that at small values of parameter $\delta$ the output signal changes linearly with stress or difference between its principal components that lie in the plane normal to the wave vector.

Sample displacement relatively to the light beam along the $x$ coordinate was linked to one of the graph plotter inputs. A signal from the amplifier came to another input. This made it possible to automatically register the birefringence distribution. In such a way we measured the birefringence distribution and (related to it) stress along one of the sample coordinates. The stress magnitude was determined using the measuring system calibration from an equivalent sample where a uniform stress influencing a preset value has been produced.

\section{Results and discussion}

Shown in Fig. 2 is the experimentally determined distribution of the difference $\left(\sigma_{x}-\sigma_{y}\right)$ between the principal components of stress (that produce anisotropy of dielectric properties in the $x-y$ plane ) for three different treatments of the sample surface. It should be noted, first of all, that the maximal stress values in Fig. 2 are almost an order of magnitude less than those at which the sample were a quarter-wave plate (and, accordingly, a signal from the circular component of polarized light were maximal). In this case the noise level of the measuring system was from two to three orders of magnitude less than the signals corresponded to the stress values presented in Fig. 2.

One can see from the Fig. 2 that the reason for stress appearance in the crystal bulk is a near-surface layer. Even when markings from mechanical treatment have been removed from the sample surface to the greatest possible extent, a stress in this layer was still observed (curve 1). Its appearance was less pronounced as compared to curves 2 and 3. The only reason for this was that the stress value in the near-surface layer was comparable to the residual stress in the sample. Our investigations have established

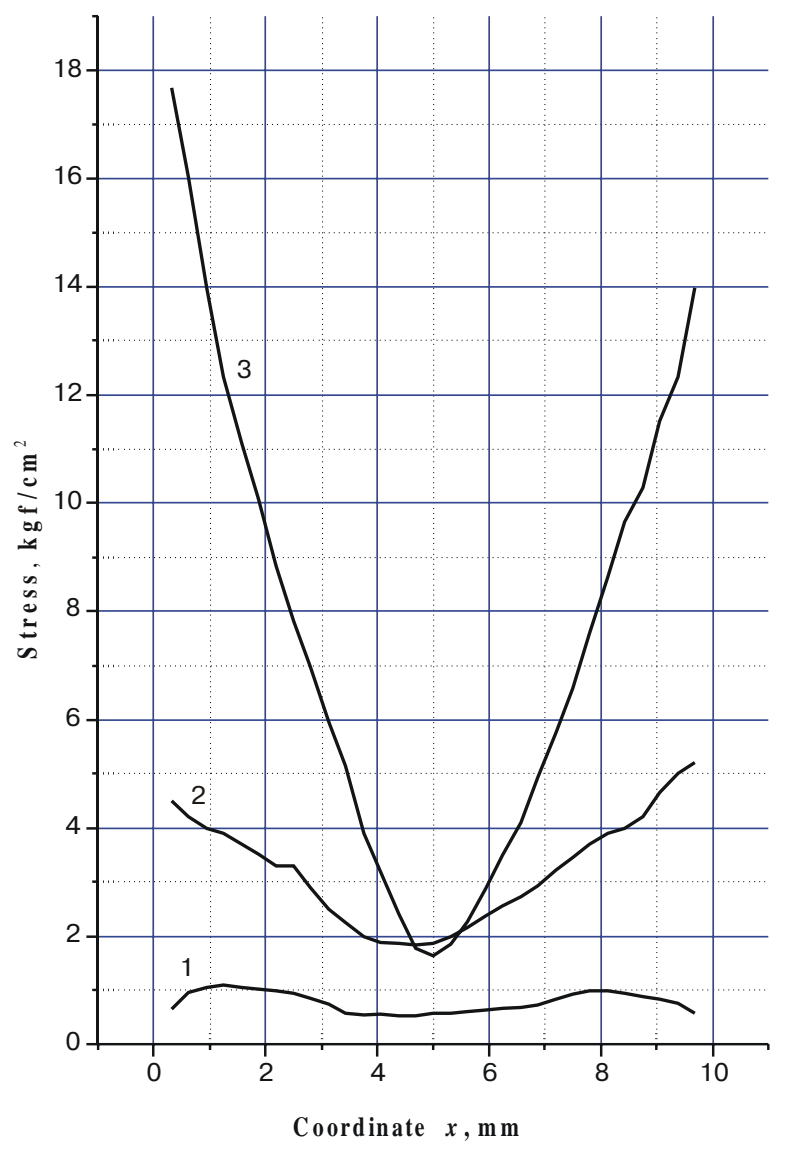

Fig. 2. Distribution of differences between principal stress components, $\sigma_{x}-\sigma_{y}$, along the coordinate $x$ in the sample whose all side faces have been: etched (1); polished with diamond paste (grain size of $2 \mathrm{~mm}$ ) (2); lapped with a powder with grain sizes of $40 \mu \mathrm{m}(3)$.

SQO, 4(1), 2001 


\section{B.K. Serdega et al.: Effect of surface condition on strain in semiconductor crystal sample}

that these stresses exist even in those initial crystals that have not been specifically doped. They are due to nonuniform distribution of crystal lattice defects.

As to the cases presented by curves 2 and 3, one can state with assurance that the nature of corresponding stresses is related to dislocations that appeared as a result of mechanical treatment of the crystal. It was established in [4] that the dislocation density depends on both the abrasive grain size and forces applied during lapping. It may be as high as $3 \cdot 10^{7} \mathrm{~cm}^{-2}$. Spatial distribution of dislocations along the normal to the surface is nonuniform. Their density goes down as the distance from the surface increases; it becomes zero at a depth comparable to the grain size. Even in the most favorable for dislocation detection case (when we used for surface treatment a powder with grain diameter of about $40 \mu \mathrm{m}$ ), direct probing of the near-surface layer using the optical-polarization technique seems to be conjectural. However, one can see from Fig. 2 that manifestation of this layer through a macroscopic strain of the sample is quite pronounced. Moreover, an additional measurement has established that the sign of bulk strain (stretching) correlates well with both the sign and nature of the near-surface strain (compression). It is known [5] that, according to the condition of sample equilibrium, there exists an unambiguous correspondence between the magnitudes, as well as signs, of strains for the near-surface layer and bulk. This means that the sign of surface strain has to be opposite to that of bulk strain, and stresses in the near-surface layer are much greater than those in the bulk. Their magnitudes depend on the sample sizes (all other factors being equal) and, as it has been done in [6] for other configuration, can be calculated. However, taking into consideration that in this case one can obtain only numerical (and not analytical) results, it seems reasonable to make such calculations independently for every individual case.

\section{Conclusions}

We have demonstrated unique potentialities of the optical-polarization technique (improved by the use of polarization modulation) and showed how changes in surface properties lead to appearance of anisotropy of the dielectric properties in the bulk. However, not only such strong actions as those used by us can be detected with the polarization-modulation technique. Taking into account that the range of stresses that can be measured by our method spans several orders of magnitude, it is possible to detect practically any surface reconstruction and relaxation of its properties.

\section{References}

1. E.F. Venger, E.V. Nikitenko, B.K. Serdega, Study of properties of radiation polarization modulator in polarimetric optical systems (in Russian) // Optoelektronika i Poluprovodnikovaya Tekhnika, N 33, pp. 102-108 (1998).

2. W.C. Dash, R. Newman, Intrinsic optical absorption in single crystal Ge and Si at 77 and $300 \mathrm{~K} / /$ Phys. Rev. 99(4), pp. 1151-1155 (1955).

3. S.N. Jasperson, S.E. Schnatterly, An improved method for high reflectivity ellipsometry based on a new polarization modulation technique // Rev. Sci. Instr. 40(6), pp. 761-767 (1969).

4. M.M. Novikov, V.M. Tsmots', Z.F. Ivasiv, Ya.L. Zayats', V.S. Shtym, Detection of non-growth dislocations in singlecrystalline silicon from measurement of magnetic susceptibility (in Ukrainian) // Ukr. Phys. Zhurn. 41(11-12), pp. 11231126 (1996).

5. A.D. Kovalenko, Introduction to Thermoelasticity (in Russian), Naukova Dumka, Kiev (1965).

6. V.Ya. Kiblyk, B.A. Novomins'kyi, M.L. Stakhurs'kyi, V.F. Trunevych, V.K. Khruts'kyi, Spatial distribution of stresses in an insulator-semiconductor system (in Ukrainian) // Ukr. Phys. Zhurn. 41(11-12), pp. 1127-1130 (1996). 